\title{
Atomic hyperfine resonances in a magnetic quadrupole field
}

\author{
Shahpoor Saeidian* \\ Physikalisches Institut, Universität Heidelberg, \\ Philosophenweg 12, 69120 Heidelberg, Germany \\ Igor Lesanovsky $\dagger$ \\ Institut Quantenoptik und Quanteninformation, \\ Institut für Theoretische Physik, Universität Innsbruck, \\ Technikerstrasse 21 a, A-6020 Innsbruck, Austria \\ Peter Schmelchent \\ Physikalisches Institut, Universität Heidelberg, \\ Philosophenweg 12, 69120 Heidelberg, Germany and \\ Theoretische Chemie, Institut für Physikalische Chemie, \\ Universität Heidelberg, INF 229, 69120 Heidelberg, Germany
}

(Dated: November 15, 2018)

\begin{abstract}
The quantum resonances of an atom possessing a single valence electron which shows hyperfine interaction with the nucleus is investigated in the presence of a three dimensional magnetic quadrupole field. Particular emphasis is put on the study of the interplay of the hyperfine and quadrupole forces. Analyzing the underlying Hamiltonian a variety of symmetries are revealed which give rise to a two-fold degeneracy of the resonance energies. Our numerical approach employs the complex scaling method and a Sturmian basis set. Several regimes and classes of shortlived and long-lived resonances are identified. The energies and decay widths of the resonances are characterized by their electronic and nuclear spin properties.
\end{abstract}

PACS numbers: 32.60.+i,33.55.Be,32.10.Dk,33.80.Ps

*s`saeid@physi.uni-heidelberg.de

†'Igor.Lesanovsky@uibk.ac.at

Peter.Schmelcher@pci.uni-heidelberg.de 


\section{INTRODUCTION}

Ultracold atomic gases offer a wealth of opportunities for studying quantum phenomena at mesoscopic and macroscopic scales [1, 2, 3, 4, 5]. The majority of experiments in the ultra-low temperature regime have so far been performed with alkali atoms. Their groundstate electronic structure is characterized by the fact that all electrons but one occupy closed shells. The latter, the so-called valence electron, is situated in the s-orbital of the outermost shell. The coupling of the nuclear spin $I$ to the total angular momentum of the active electron via the hyperfine interaction causes a splitting of the electronic energy levels into several branches which can be characterized by the quantum number of the total spin $F$. Due to a vanishing orbital angular momentum this hyperfine interaction consist exclusively

of the coupling of the electronic spin $S=\frac{1}{2}$, to the nuclear spin $I$. This leads to the two possibilities $F=I \pm S$.

Inhomogeneous magnetic field configurations represent a key component for the control of the motion of cold atoms, specifically for their trapping. The underlying Zeeman interaction occurs due to coupling of the total magnetic moment of the atom to the external magnetic field. Quantum states and particularly resonances of atoms in inhomogeneous field configurations are therefore of immediate interest to cold atomic physics in general and have been investigated in the past by several groups. This includes the quadrupole field [6, 7], the wire trap [8, 9, 10, 11] and the magnetic guide and Ioffe trap [12, 13, 14, 15, 16]. In these traps we typically encounter quantum resonances with a certain lifetime instead of stationary 'stable' quantum states. Very recently [17] a new class of short-lived resonances possessing negative energies for the case of the $3 \mathrm{~d}$ magnetic quadrupole field was found. These resonances originate from a fundamental symmetry of the underlying Hamiltonian. In contrast to the positive energy resonances, they are characterized by the fact that the atomic magnetic moment is aligned antiparallel to the local direction of the magnetic field, and their lifetimes decrease with increasing total magnetic quantum number.

In all of the above investigations it is assumed that the hyperfine interaction is much stronger than the interaction with the external inhomogeneous magnetic field and therefore the electronic and nuclear angular momenta firstly provide a total angular momentum which then interacts with the magnetic field. This physical picture truly holds for (alkali) atoms in their electronic ground state and macroscopic as well as microscopic (atom chip) gradient 
fields. The atom is consequently treated as a point particle with the total angular momentum $F$. In the present work we study the case for which both interactions, the hyperfine and the field interaction, have to be taken into account on equal level for the description of the neutral atoms in the field. This case is primarily of principal interest but is expected to describe the magnetized hyperfine properties of systems with very small hyperfine interactions and/or strong gradient fields, such as electronically excited atoms. In the latter case an admixture of different hyperfine states $F$ due to the field interaction has to be expected. In ref. [18] the possibility to tune the hyperfine splitting by dressing the electronic energy levels by a microwave has been demonstrated. Hence, by this method it is thinkable to achieve a scenario in which the magnetic and the hyperfine-interaction become comparable even for ground state atoms.

We focus on atoms possessing a single active valence electron with spin $S=\frac{1}{2}$ and a nucleus with spin $I=\frac{3}{2}$ in a three dimensional magnetic quadrupole field. In detail we proceed as follows. Section II contains the derivation of the underlying Hamiltonian. Our computational method to calculate the resonance energies and lifetime is outlined in section III. Section IV contains a presentation and discussion of our results. We conclude with a summary in section $\mathrm{V}$.

\section{HAMILTONIAN}

Taking into account the hyperfine interaction, the Hamiltonian describing the motion of an atom with mass $M$ and electronic and nuclear spin $S$ and $I$, respectively, reads in a magnetic quadrupole field

$$
H=\frac{\mathbf{p}^{2}}{2 M}+H_{H F}+H_{B}
$$

where $H_{H F}$ describes the hyperfine interaction between the outermost single valence electron and the nucleus

$$
H_{H F}=A \mathbf{I} \cdot \mathbf{S}
$$

Here $A$ is a constant which for an s-electron is given by [19]

$$
A=\frac{2}{3} \frac{\mu_{0}}{\hbar^{2}} g_{e} g_{n} \mu_{B} \mu_{N}\left|\psi_{s}(0)\right|^{2}
$$

$g_{e}$ and $g_{n}$ are the $g$-factors of the electron and the nucleus, respectively, and $\psi_{s}(0)$ is the

value of the valence s-electron wave function at the nucleus. $\mu_{B}=\frac{e \hbar}{2 m_{e}}$ and $\mu_{N}=\frac{e \hbar}{2 m_{p}}$ 
are the Bohr magneton for the electron and the proton, respectively. $H_{B}$ accounts for the interaction of the magnetic moment of the electron and the nucleus with the magnetic field

$$
H_{B}=\mu_{B} g_{e}(\mathbf{S}+\alpha \mathbf{I}) \cdot \mathbf{B}
$$

with

$$
\alpha=-\frac{\mu_{N} g_{n}}{\mu_{B} g_{e}} \approx-\frac{m_{e}}{m_{p}} .
$$

The vector of the three-dimensional quadrupole magnetic field is given by $\mathbf{B}(\mathbf{r})=$ $b(x, y,-2 z)$. By substituting equations (2-5) into equation (1) and performing the scale transformation

$$
\bar{p}_{i}=\left(2 M b \mu_{B} g_{e}\right)^{-1 / 3} p_{i} \quad \text { and } \quad \bar{x}_{i}=\left(2 M b \mu_{B} g_{e}\right)^{1 / 3} x_{i}
$$

we obtain

$$
\begin{aligned}
H= & \frac{1}{2}\left[\mathbf{p}^{2}+x\left(S_{x}+\alpha I_{x}\right)+y\left(S_{y}+\alpha I_{y}\right)\right. \\
& \left.-2 z\left(S_{z}+\alpha I_{z}\right)+\beta \mathbf{I} \cdot \mathbf{S}\right]
\end{aligned}
$$

with $\beta=\frac{A}{b \mu_{B} g_{e}}$, where the bar have been omitted. The energy is now measured in units of $\frac{1}{M}\left(2 M b \mu_{B} g_{e}\right)^{2 / 3}$. For convenience and in anticipation of the forthcoming discussion we transform the Hamiltonian to a spherical coordinate system, i.e. $(x, y, z) \rightarrow(r, \theta, \phi)$. Writing the momentum operator explicitly and using atomic units we obtain

$$
\begin{aligned}
H= & \frac{1}{2}\left[-\frac{\partial^{2}}{\partial r^{2}}-\frac{2}{r} \frac{\partial}{\partial r}+\frac{L^{2}}{r^{2}}+r \sin \theta \cos \varphi\left(S_{x}+\alpha I x\right)\right. \\
& +r \sin \theta \sin \varphi\left(S_{y}+\alpha I_{y}\right)-2 r \cos \theta\left(S_{z}+\alpha I_{z}\right) \\
& +\beta \mathbf{I} \cdot \mathbf{S}] .
\end{aligned}
$$

Exploring the symmetries of the Hamiltonian we find 16 discrete symmetry operations and a continuous symmetry generated by $J_{z}$, which is the z-component of the total angular momentum of the atom.

The Hamiltonian (6) basically differs from the Hamiltonian (4) in ref. [7] by the additional hyperfine interaction term. This term does not introduce new symmetries and degeneracies except that all symmetry operations found in ref.[7] have now to be generalized to the case of the additional presence of the nucleus angular momentum. For a discussion of degeneracy and symmetries in more details, we refer the reader to ref.[7]. 


\section{NUMERICAL APPROACH}

The Hamiltonian (6) does not support bound states. Its continuous spectrum is characterized by resonances which are localized in space (at $t=0$ ). The time evolution of these states is given by

$$
\psi_{R}(t)=e^{\frac{-i E t}{\hbar}} \psi_{R}(0)
$$

where $E$ is complex

$$
E=\varepsilon-i \frac{\Gamma}{2}
$$

Here $\varepsilon$ and $\Gamma$ are the energy and decay width of the resonance, respectively. Because of the imaginary part $-i \frac{\Gamma}{2}$ the resonances decay exponentially with a lifetime of $\tau=\Gamma^{-1}$. In order to calculate the energies and decay widths of the scattering wave functions we employ the complex scaling method (see ref.[20] and references therein). The complex scaling transformation only affects the continuum, while the bound states do not change their positions. The continuum states are rotated around the corresponding threshold into the lower half complex energy plane. Eigenvalues belonging to resonances, once revealed, maintain their positions.

Since resonance states of the complex scaled Hamiltonian are square integrable the linear variational principle can be applied. Expanding the wavefunction in a set of basis functions and calculating the expansion coefficients results in a large-scale algebraic generalized eigenvalue equation which can be solved by employing a Krylov space method [21]. The basis set must be chosen in such a way that the exact wave function can be approximated to a sufficient degree of accuracy by as small as possible number of functions. Therefore the form

of the basis set must be adapted to the geometry and the symmetries of the system. We found a so-called Sturmian basis set of the form

$$
\left|n, l, m_{i}, m_{s}\right\rangle_{m}=R_{n}^{(\zeta)}(r) Y_{l}^{m-m_{i}-m_{s}}(\theta, \varphi) \chi^{i}\left(m_{i}\right) \chi^{s}\left(m_{s}\right)
$$

suitable for our purposes. Here the functions $Y_{l}^{m}$ are the spherical harmonics. For fixed $m$ the linear variational combination of the basis functions $\psi_{n, l, m_{i}, m_{s}}^{m}$, yields, per construction, eigenstates of the Hamiltonian and $J_{z}$ simultaneously. For expanding the radial part we take the non-orthogonal set of functions

$$
R_{n}^{(\zeta)}(r)=e^{-\frac{\zeta r}{2}} L_{n}(\zeta r)
$$


where $L_{n}(x)$ are the Laguerre polynomials. The free parameter, $\zeta$, has the dimension of an inverse length and can be tuned to improve the convergence behavior in different regions of the energy spectrum. It should be chosen such that $1 / \zeta$ corresponds to the typical length scale of the states to be approximated. Using the basis set (10) all matrix elements of the Hamiltonian(7) can be calculated analytically.

\section{RESULTS}

Let us now discuss the results we obtained while studying an atom with hyperfine interaction in a magnetic quadrupole field. We present the resonance energies and decay widths for different values of the field gradient. The resulting spectrum consist of several well-separated parts. Concerning the resonance positions one can distinguish three regimes, each of which reveals individual characteristics: the weak, the intermediate, and the strong gradient regimes. In the weak gradient regime, the Zeeman term $H_{B}$ is very small compared with the hyperfine interaction $H_{H F}$ and only slightly perturbs the zero-field eigenstates of $H$. In this case the atom, being primarily in its hyperfine ground state $F=I-\frac{1}{2}$, remains in this manifold and behaves approximately like a neutral particle of spin $F$ with the $g$-factor

$$
g_{f} \simeq g_{e} \frac{F(F+1)-I(I+1)+S(S+1)}{2 F(F+1)} .
$$

This regime is also called the Zeeman regime. In the intermediate gradient regime the Zeeman and the hyperfine interactions are of the same order of magnitude, and the atom in the ground state may represent a significant admixture of different hyperfine states $F$. Finally in the strong gradient regime the Zeeman term dominates the hyperfine energies at least for sufficiently large distances from the coordinate origin. In this case the spin component of the electron along the local direction of the magnetic field is almost conserved and as we will see, the resonance positions in the complex energy plane are grouped according to different values of its quantum number $m_{s}$. This regime is also called the Paschen-Back Regime.

Knowing the resonance eigenfunctions of the complex-scaled Hamiltonian one can calculate corresponding expectation values within the generalized inner c-product [20]. The expectation value which is obtained in this way is in general complex. The real part represents the average value, whereas the imaginary part can be interpreted as the uncertainty 
of our observable in a measurement when the system is prepared in the corresponding resonance state [20]. We will analyze the average values of the components of the spins which point along the local direction of the field as a function of the energy. This enable us to explain different sets of resonances in each regime. We also discuss in this section the dependence of the decay width of a resonance state on its angular momentum as well as on the field gradient.

\section{A. Resonance Positions in the Zeeman Regime}

For large values of $\beta(\beta>200)$ we are in the Zeeman regime. In Fig. 1(a-c) we present the energies and decay widths for $\beta=1000$ and $m=10$, for an atom with nuclear spin $I=\frac{3}{2}$ being in its hyperfine ground state. The resonances are localized in the negative energy region and their distribution consists of three well-separated parts. They can be classified according to the expectation value $m_{F}=\operatorname{Re}(\langle\mathbf{F} \cdot \mathbf{B} /|\mathbf{B}|\rangle)$ which is the projection of the total spin onto the local direction of the magnetic field. There are long-lived resonances which can be identified with $m_{F}=-1$, and two sets of resonances with shorter lifetimes whose spin projections are $m_{F}=0,+1$. The values of $m_{F}$ are shown in the panel $\mathrm{d}$. For $m_{F}=-1$ one observes the resonances to be located on lines with similar slopes covering the area of a right triangle in the $\epsilon-\Gamma$ plane. For $m_{F}=0$ and $m_{F}=+1$ one immediately notices that both sets are arranged on lines with an infinity and a negative slope respectively. In Fig.1(e) we present the decay width of the energetically lowest state of the long-lived resonances (the resonances with $m_{F}=-1$ ) as a function of the angular momentum $m$. The decay width decreases exponentially with increasing value of $m$. We also present the decay width of the energetically highest state of the two sets of short-lived resonances as a function of the quantum number $m$. Unlike the case $m_{F}=-1$, the decay width of resonances with $m_{F}=+1$ and $m_{F}=0$ increases with increasing angular momentum. (for an explanation of this behavior see [7]). Fig!1(f) shows the expectation value of the squared total spin, $R e\left(\left\langle\mathbf{F}^{\mathbf{2}}\right\rangle\right)$ as a function of the energy for the same parameter values. For all resonance states, the value is approximately +2 which corresponds to $F=1$ i.e. the atom behaves like a spin-1 particle.

In Fig 2 we present the lifetime of the energetically lowest long-lived resonance state $\left(m_{F}=-1\right)$ for $m=25$ as a function of the parameter $\beta$. The lifetime decreases as the 

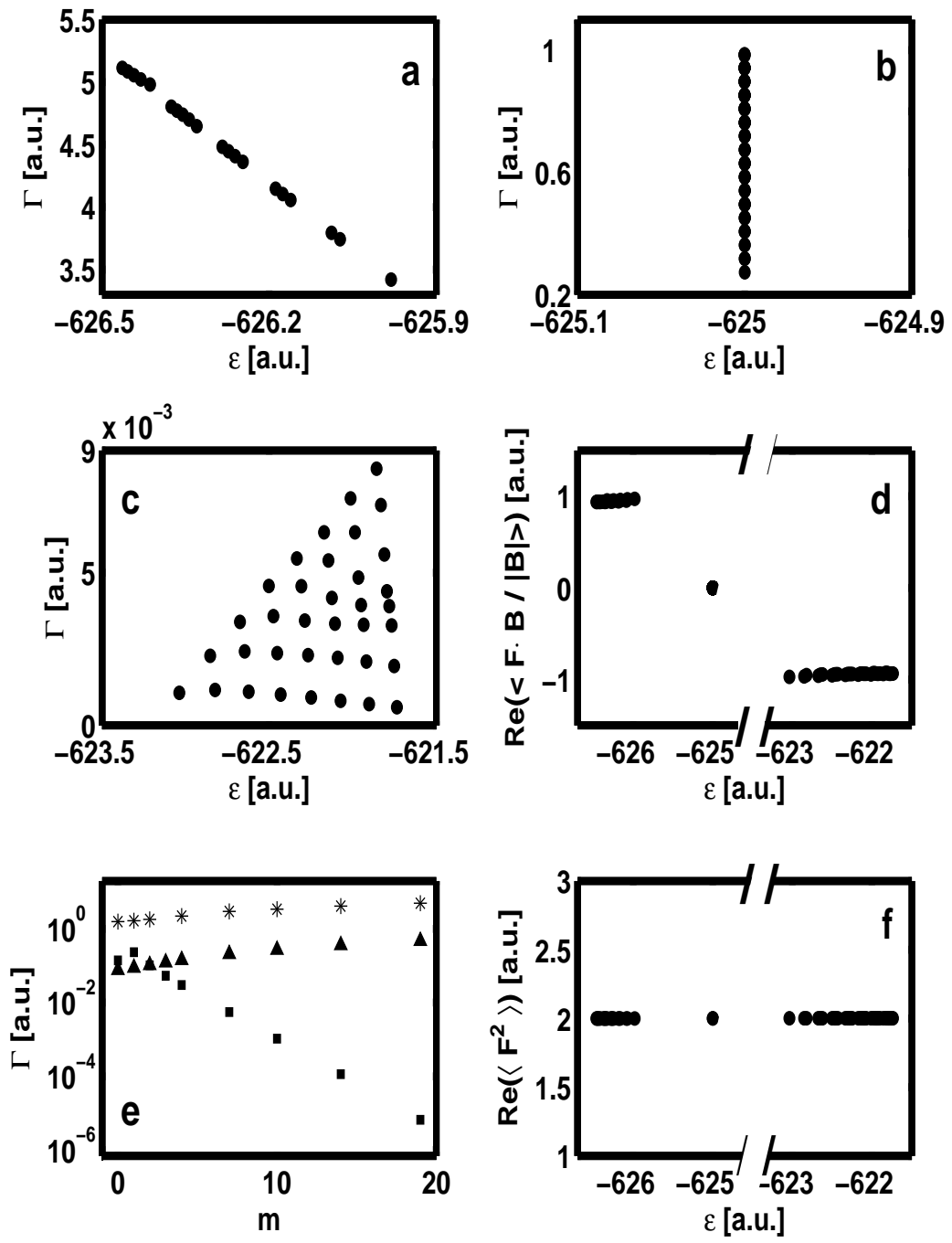

FIG. 1: Decay width and energies of the resonances for $\beta=1000, m=10$ for (a) $m_{F}=1$, (b) $m_{F}=0$ and (c) $m_{F}=-1$. (d) Expectation values of the total spin component along the local direction of the magnetic field for the three sets of resonances. (e) (ם) The decay width of the energetically lowest state with $m_{F}=-1,(\mathbf{\Delta})$ the decay width of the energetically highest state with $m_{F}=0,(*)$ the decay width of the energetically highest state with $m_{F}=+1$ as a function of the quantum number m. (f) The expectation value of the total spin squared as a function of the energy. 


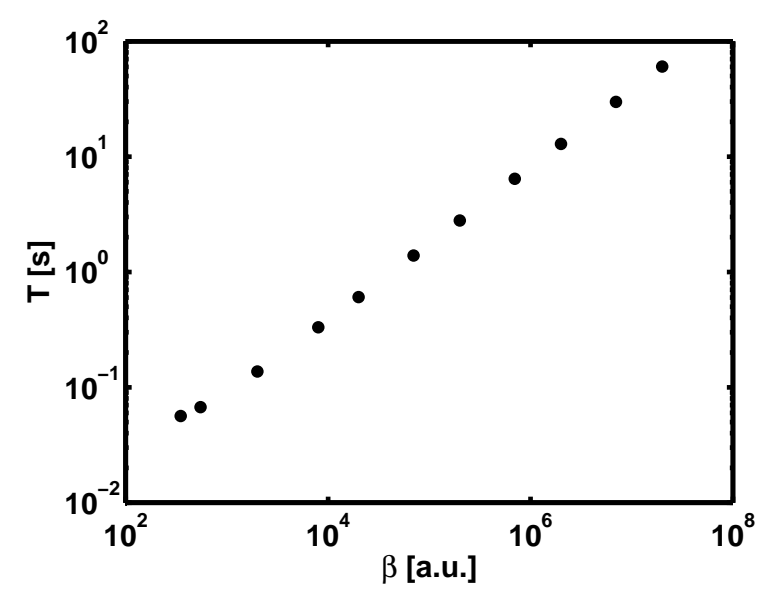

FIG. 2: The lifetime of the energetically lowest long-lived resonance state for $m=25$ as a function of the parameter $\beta$.

hyperfine parameter $\beta$ decreases, i.e. the gradient field $\mathrm{b}$ increases. Performing a line fit we find the dependence $T[s] \approx 8.3 \times 10^{-4} \beta^{2 / 3}[$ a.u. $]$.

\section{B. Resonance Positions in the Intermediate Regime}

Let us now focus on the intermediate regime covering the values $0.2<\beta<200$, where the Zeeman and hyperfine interactions become comparable. We observe three different types of behavior. For $40<\beta<200$, an atom being in the hyperfine ground state, still behaves approximately like a spin-1 particle. The resonance spectrum is localized in the negative energy domain and consists of three well-separated parts to which we can assign the values $m_{F}=-1,0,+1$. For the two short-lived states, following the same reasoning as above, the width increases when $m$ increases, while for the long-lived states it decreases. However in contrast to the Zeeman regime (see Fig.2), the lifetime of the long-lived states $\left(m_{F}=-1\right)$ increases when the hyperfine parameter $\beta$ decreases, i.e. the field gradient $\mathrm{b}$ increases (see Fig (3). For $\beta<40$ this value decreases very rapidly when $\beta$ decreases. In this case the resonance states which correspond to higher hyperfine levels $F=2$ are more stable, and are localized in the positive region of the spectrum. Fig 4 (a) shows the decay widths and energies of the resonances possessing positive energies for $\beta=19$. We observe four different

curved and triangular shaped regions with distinct classes of resonances. In Fig 4(b) we present the expectation values of the squared total spin, $\operatorname{Re}\left(\left\langle\mathbf{F}^{2}\right\rangle\right)$, of these resonances as 


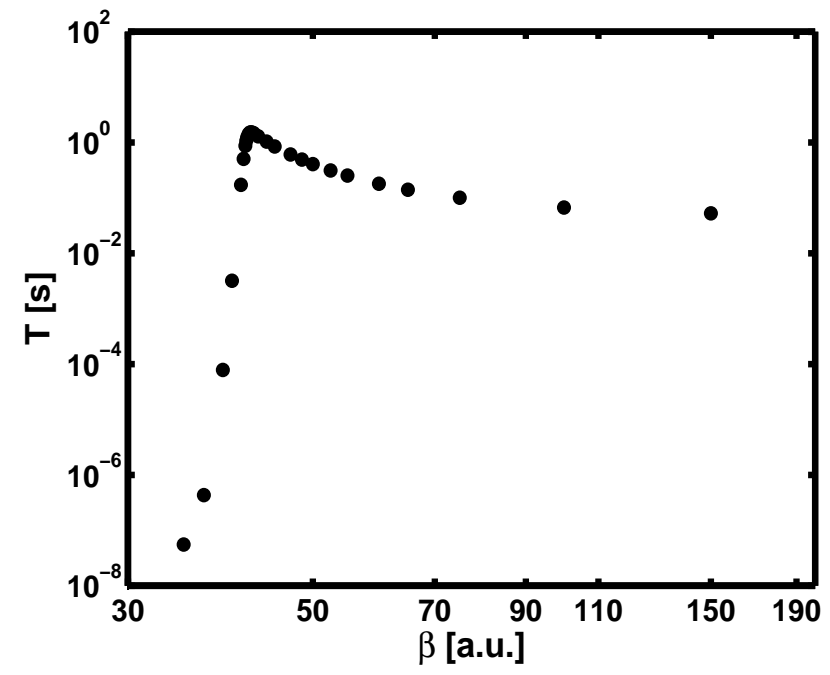

FIG. 3: The lifetime of the energetically lowest long-lived resonance state for $m=25$ as a function of the parameter $\beta$.

a function of the energy. The values range from approximately 5.5 to 6.0 , correspond to $F \approx 2.0$. Fig 4 (c) shows the expectation values of the total spin component along the local direction of the field for the resonances of Fig 4(a). The resonances are divided into four well-separated parts corresponding to $m_{F}=+2,+1,0,-1$. Resonances with $m_{F}=-2$ have very short lifetime and are not shown. For $\beta<3$ the non-conservation of $F$ becomes even more explicit, see Fig.5(a) for $\beta=1$. In Fig. 5(b,c) we present the respective decay widths and energies. The resonances which are localized in the negative energy region have short lifetimes and are divided into four subgroups. Each group lies on a line with negative slope, the four slopes being very similar. Moreover, the resonances investigated form subgroups on these lines. The positive energy resonances have much longer lifetimes and cover an area of approximately triangular shape in an irregular manner, i.e. no pattern is visible. In Fig 5 (d) the corresponding expectation values of the electronic spin component along the local direction of the magnetic field, $\operatorname{Re}(\langle\mathbf{S} \cdot \mathbf{B} /|\mathbf{B}|\rangle)$ is shown. For the negative energy resonances this value is approximately -0.5 indicating that the spin is aligned opposite to the local direction of the magnetic field, while for positive energies the spin is parallel to the magnetic field. Fig 5 (e) shows the expectation values of the nuclear spin component along the local direction of the field. In the negative energy domain of the spectrum the pattern divides into four parts with values $m_{I}=-\frac{3}{2},-\frac{1}{2},+\frac{1}{2},+\frac{3}{2}$, while in the positive energy region, the pattern is strongly disturbed and $m_{I}$ is not conserved. Fig.5(f) presents the lifetime of 

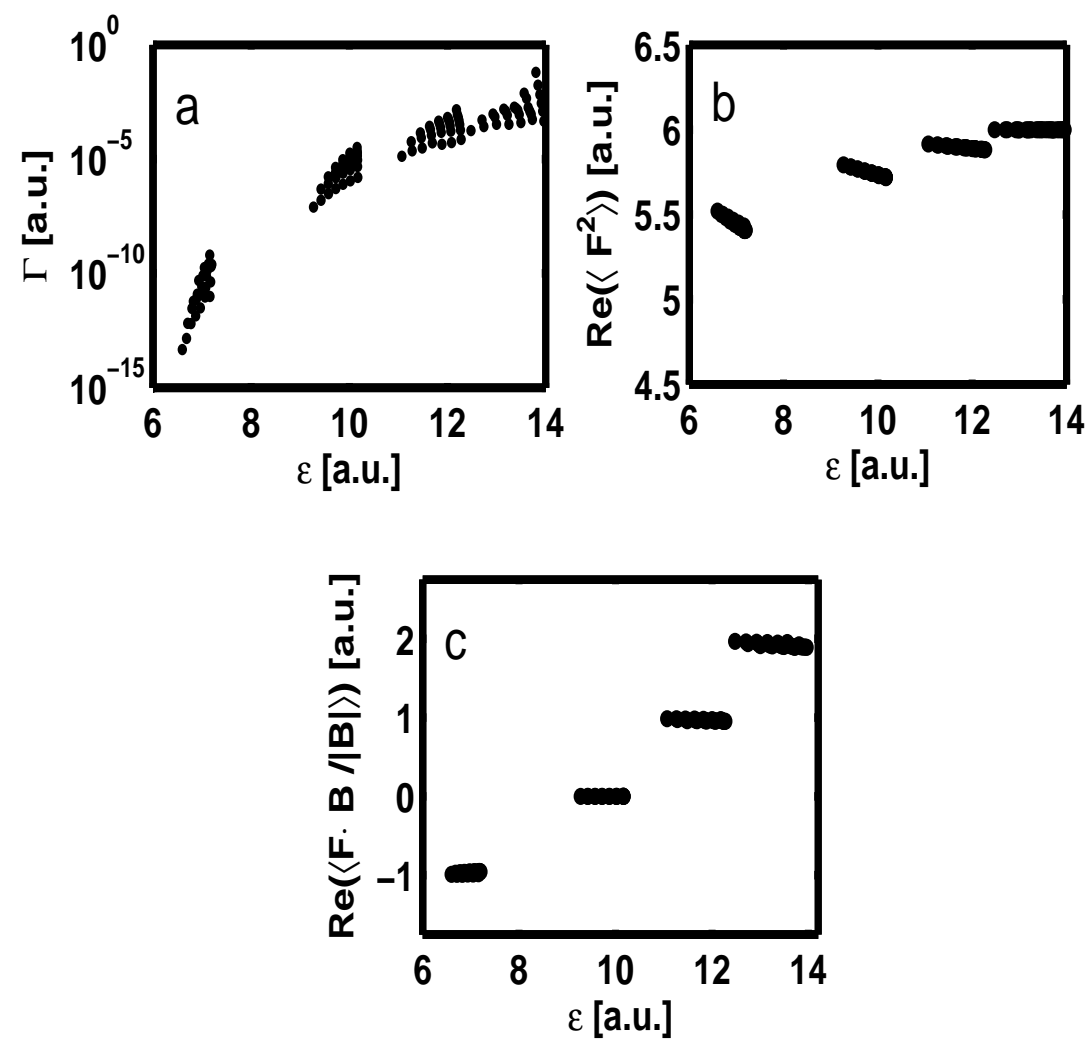

FIG. 4: (a) Decay width and energies of the long-lived resonances with higher hyperfine quantum number $F=2$ for $\beta=19$ and $m=25$. (b) The expectation value of the total spin squared as a function of the energy. (c) Expectation values of the total spin component along the local direction of the magnetic field as a function of the energy.

the energetically lowest long-lived state as a function of $\beta$. This value increases when $\beta$ decreases, i.e. the gradient field b increases. Our results show that for the short-lived states, i.e. the negative energy resonance states, the width increases when $m$ increases, while for the long-lived states, i.e. the positive energy resonance states, it decreases.

\section{Resonance Positions in the Hyperfine Paschen-Back Regime}

The hyperfine Paschen-Back regime includes $\beta<0.2$. In case the Zeeman term dominates the hyperfine energy, it is natural to decompose the Hamiltonian according to $H=H_{0}+H_{1}$

where $H_{0}=\frac{1}{2}\left(\mathbf{p}^{\mathbf{2}}+\mathbf{x S}_{\mathbf{x}}+\mathbf{y} \mathbf{S}_{\mathbf{y}}-\mathbf{2} \mathbf{z} \mathbf{S}_{\mathbf{z}}\right)$ describes the motion of a spin $\frac{1}{2}$ particle in the magnetic field, and $H_{1}=\alpha\left(x I_{x}+y I_{y}-2 z I_{z}\right)+\beta \mathbf{I} \cdot \mathbf{S}$ perturbs the eigenstates of $H_{0}$. The spectrum consists of two parts: again we have one set of resonances localized in the negative 

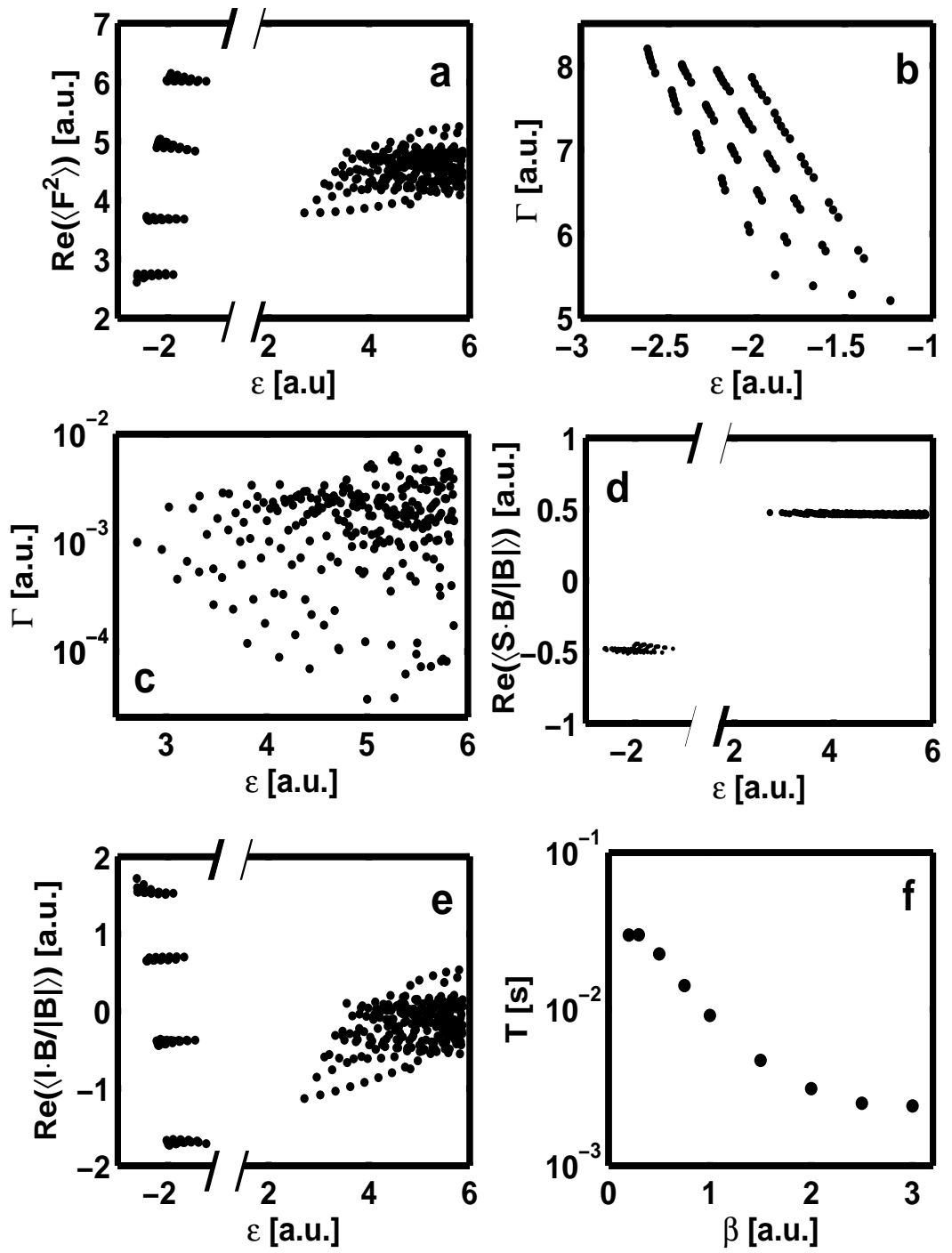

FIG. 5: (a) The expectation value of the total spin squared as a function of the energy for $m=10$ and $\beta=1.0$. Decay width and energies of the resonances for (b) $m_{S}=-\frac{1}{2}$, (c) $m_{S}=+\frac{1}{2}$. (d) Expectation values of the electronic spin component along the local direction of the magnetic field. (e) Expectation values of the nuclear spin component along the local direction of the magnetic field. (f) The lifetime of the energetically lowest resonance state of the positive energy domain of the spectrum as a function of $\beta$. 
energy region with short lifetimes and a second set localized in the positive energy domain possesses much larger lifetimes. In Fig,6(a,b) we present the energies and decay widths of resonances for $\beta=0.1$ and $m=10$. The negative energy resonances are arranged on lines with a negative slope. The positive energy resonances cover an area of triangular shape, some of them being located on straight lines. Fig 6(c) shows the corresponding expectation value $\operatorname{Re}\left(\left\langle\mathbf{F}^{2}\right\rangle\right)$ of the squared total spin. This value is almost 4.5 which again indicates that the total spin quantum number $F$ is not conserved. In Fig 6 (d) the respective expectation value $\operatorname{Re}(\langle\mathbf{S} \cdot \mathbf{B} /|\mathbf{B}|\rangle)$ of the electronic spin component which points along the local direction of the magnetic field is presented. For the positive energy resonances this value is approximately +0.5 indicating that the spin is aligned parallel to the local direction of the magnetic field while for negative energy resonances it is antiparallel. The nuclear spin component along the local field is not conserved, however it is instructive to consider

its average value, $\operatorname{Re}(\langle\mathbf{I} \cdot \mathbf{B} /|\mathbf{B}|\rangle)$, as a function of the energy. For large values of $m$ (see Fig $6(\mathrm{e})$ for $m=10$ ) the resonances are grouped into four regular subparts for both the positive and negative energy domain.

We remark that the decay widths of the positive energy resonances show again an exponential decaying behavior as a function of the quantum number $m$ whereas the decay widths of the negative energy resonance states increases with increasing $m$. The lifetime of the energetically lowest long-lived resonances for $m=25$ as a function of $\beta$ can be fitted according to $T[s] \approx 1.1 \times 10^{-1} \beta^{2 / 3}[$ a.u. $]$.

\section{SUMMARY}

We have investigated the resonant quantum properties of an atom with a single active valence electron taking into account its hyperfine structure $\left(I=\frac{3}{2}\right)$ in a $3 \mathrm{~d}$ magnetic quadrupole field.

We have calculated and analyzed the energies and decay widths of the resonance states of the Hamiltonian employing the complex scaling approach and a Sturmian basis set. With respect to the resonance position one can distinguish essentially three regimes. In the weak gradient regime, the Zeeman term is very small compared with the hyperfine interaction and only slightly perturbs the zero-field eigenstates of the Hamiltonian. In this case the atom being primarily in its hyperfine ground state behaves approximately like a particle of 

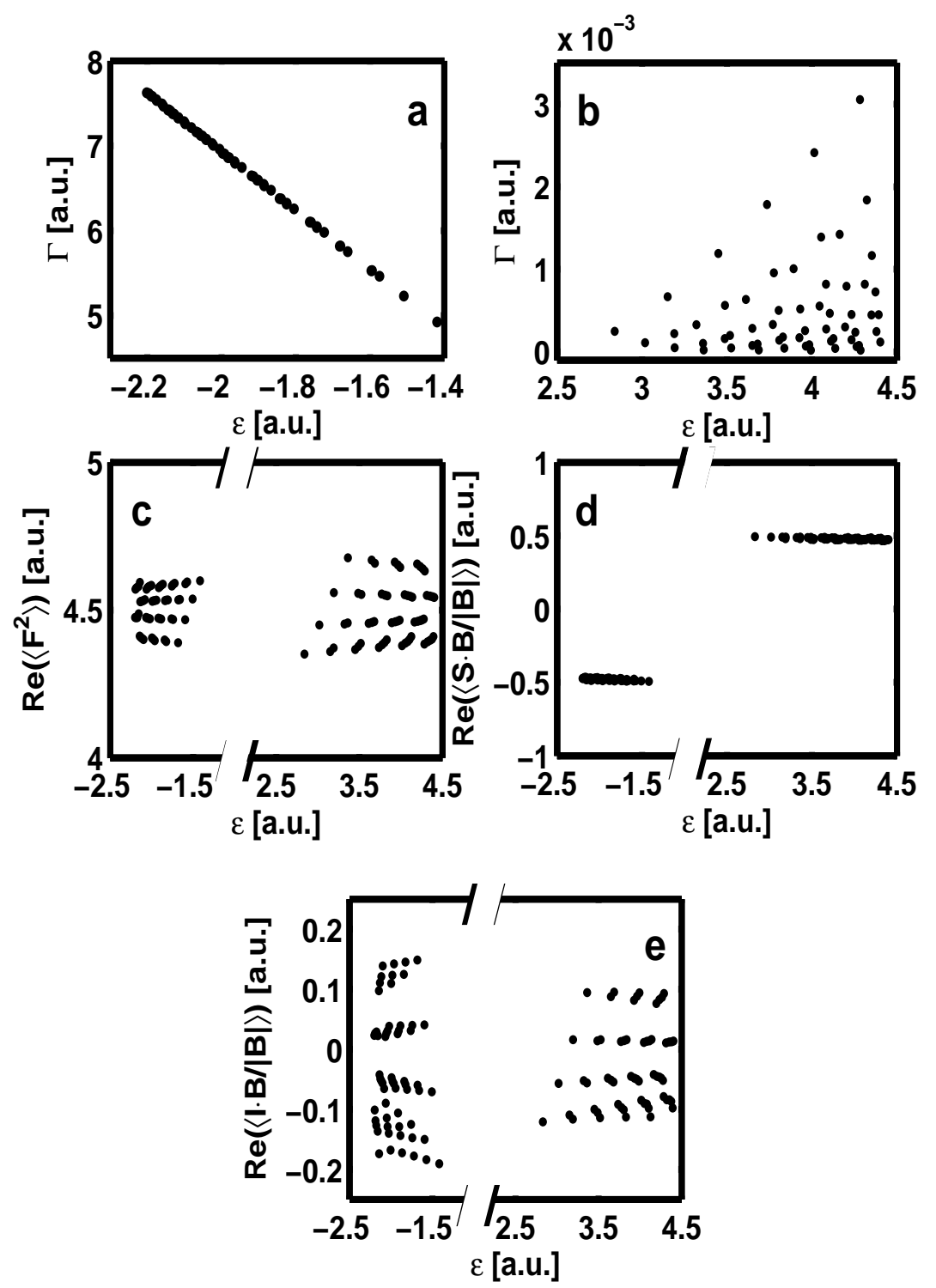

FIG. 6: Decay width and energies of the resonances for $\beta=0.1, m=10$ for (a) $m_{S}=-\frac{1}{2}$, (b) $m_{S}=+\frac{1}{2}$. (c) The corresponding expectation value of the total spin squared as a function of the energy. (d) Expectation values of the electronic spin component along the local direction of the magnetic field for the two sets of resonances. (e) Expectation values of the nuclear spin component along the local direction of the magnetic field.

spin 1, and the resonance states are grouped into three well-separated parts, corresponding to three different directions of the total spin with respect to the local direction of the field. The resonances for which the total spin is antiparallel to the field $\left(m_{F}=-1\right)$ possess the 
largest lifetimes. As the total angular momentum of the atom increases, the decay width of a short-lived resonance state increases while for a long-lived resonance state it decreases. Our results for the long-lived resonances show that as the scaled hyperfine parameter $\beta$ increases so does the lifetime.

In the intermediate regime the Zeeman and hyperfine interactions are comparable. For a sufficiently weak field gradient the atom in the hyperfine ground state behaves approximately like a spin-1 particle, and the resonances are arranged somehow similar to the Zeeman regime but in contrast to the Zeeman regime its long-lived resonance states become more stable when the gradient field increases and/or $\beta$ decreases. For stronger field gradients and/or smaller values of the parameter $\beta$ the lifetime decreases rapidly. In this case resonances which correspond to the higher hyperfine level, $F=2$, are more stable. For even smaller values of $\beta$, the non-conservation of $F$ becomes remarkable. In this case the electronic spin component along the local direction of the magnetic field is almost conserved. A resonance which is localized in the negative energy region of the spectrum possesses a short lifetime. In such a state the electronic spin is aligned opposite to the local direction of the magnetic field. The positive energy resonances possess a much longer lifetime with their electronic spin being parallel to the magnetic field. For a short-lived state the width increases with increasing $m$, while for a long-lived state it decreases. For the latter our results show that the lifetime of this state increases with decreasing values of $\beta$.

In the strong gradient regime, the Zeeman term dominates the hyperfine energy. Here the component of the electronic spin along the local direction of the field is almost conserved. The energy spectrum is arranged into two disconnected parts each of which contains exclusively resonance states with negative and positive energies. The former are of short-lived character and the electronic spin is antiparallel to the local direction of the magnetic field. The latter possess much longer lifetimes and the spin of the electron is parallel to the field. As the total angular momentum of the atom increases, the decay width of a negative energy resonance state increases while for a positive energy resonance state it decreases. Similar to the weak gradient regime the lifetime of a positive energy resonance state, decreases with decreasing $\beta$.

S.S. acknowledges financial support by the Ministry of Science, Research and Technology 
of Iran.

[1] C.J. Pethick and H. Smith, Bose-Einstein Condensation in Dilute Gases, Cambridge University Press (2002)

[2] L. Pitaevskii and S. Stringari, Bose-Einstein Condensation, Oxford Science Publications (2003)

[3] W. Ketterle, D.S. Durfee and D.M. Stamper-Kurn, Proceedings Enrico Fermi summer school on Bose-Einstein condensation, Varenna, Italy (1998)

[4] A. Leggett, Rev. Mod. Phys. 73, 307 (2001)

[5] F. Dalfovo, S. Giorgini, L.P. Pitaevskii and S. Stringari, Rev. Mod. Phys. 71, 463 (1999)

[6] T. H. Bergeman et al, J. Opt. Soc. Am. B, 2249 (1989)

[7] I. Lesanovsky and P. Schmelcher, Phys.Rev. A 71, 032510 (2005)

[8] K. Berg-Sorensen et al, Phys. Rev. A 53, 1653 (1996)

[9] L. Vestergaard Hau, J.A. Golovchenko, and M. Burns, Phys. Rev. Lett. 75, 1426 (1995)

[10] J. P. Burke, Jr., Chris H. Greene, and B. D. Esry, Phys. Rev. A 54, 3225 (1996)

[11] R. Blümel,K. Dietrich, Phys.Rev. A 43, 22 (1991)

[12] E. A. Hinds and C. Eberlein, Phys. Rev. A 61, 033614 (2000)

[13] E. A. Hinds and C. Eberlein, Phys. Rev. A 64, 039902(E) (2000)

[14] R. M. Potvliege and V. Zehnlé, Phys. Rev. A 63, 025601 (2001)

[15] I. Lesanovsky and P. Schmelcher, Phys. Rev. A 70, 063604 (2004)

[16] J. Bill, M.-I. Trappe, I. Lesanovsky and P. Schmelcher, Phys. Rev. A 73, 053609 (2006)

[17] S. Saeidian, I. Lesanovsky and P. Schmelcher, Phys. Rev. A 74, 065402 (2006)

[18] F. Gerbier et al, Phys. Rev. A 73, 041602R (2006)

[19] B.H.Brandsen and C.J.Joachain, Physics of Atoms and Molecules, Longman Ltd (1983)

[20] N. Moiseyev, Phys. Rep. 302, 211 (1998)

[21] D.C. Sorensen, Parallel numerical algorithms - Implicitly restarted Arnoldi/Lanczos methods for large scale eigenvalue calculations, Kluwer (Dordrecht) (1995) 